\title{
DI (TÚ) QUE COMO MARCADOR DEL DISCURSO
}

\author{
DI (TU) QUE AS DISCOURSE MARKER
}

\author{
Francisco Javier Grande Alija \\ Universidad de León \\ Grupo de investigación Grupo SinCom \\ fjgraa@unileon.es
}

Recibido: $17 / 06 / 2019$

Aceptado: 16/10/2019

\section{Resumen}

Este trabajo se centra en el estudio del comportamiento de un marcador del discurso que ha recibido poca atención, pese a que está bien representado en el uso del español coloquial: di (tú) que. El objetivo principal de este estudio es llevar a cabo una descripción de las propiedades formales, semánticas y discursivas de di (tú) que a partir de un corpus de ejemplos de textos con rasgos coloquiales. Nuestro análisis identifica tres valores básicos: intensificación, inductor de ironía e hipótesis, así como diferentes grados de gramaticalización en el proceso que va de una construcción verbal con un verbo en imperativo a un marcador del discurso.

PALABRAS CLAVE: marcadores del discurso; intensificación; hipótesis; imperativo; gramaticalización.

\begin{abstract}
This work focuses on the study of the behavior of a discourse marker that has received little attention, although it is well represented in the use of colloquial Spanish: di (tú) que. The main objective of this study is to carry out a description of the formal, semantic and discursive properties of $d i\left(t u^{\prime}\right)$ que drawn from a corpus of examples of texts with colloquial features. Our analysis identifies three basic values: intensification, irony inductor and hypothesis, as well as different degrees of grammaticalization in the process that goes from a verbal construction with a verb in imperative to a discourse marker.

KEY WORDS: discourse markers; intensification; hypothesis; imperative; grammaticalization.
\end{abstract}

Para citar este artículo / To cite this article: Grande Alija, Francisco Javier (2019): “Di (tú) que como marcador del discurso". González Sanz, Marina y Pérez Béjar, Víctor (eds.): ELUA: Macrosintaxis en construcción, Anexo VI, págs. 133-156.

Enlace/Link: http://dx.doi.org/10.14198/ELUA2019.ANEXO6.08 


\section{INTRODUCCIÓN}

Este trabajo ${ }^{2}$ pretende ser una contribución al estudio del comportamiento de un marcador del discurso en el español actual que ha recibido poca atención, pese a que está bien representado en el uso del español coloquial. Nos estamos refiriendo a la fórmula di (tú) que. Nuestro objetivo principal es llevar a cabo una descripción de sus propiedades formales, semánticas y discursivas.

Nuestro enfoque no es cuantitativo. Nos interesa algo más básico y esencial: identificar y caracterizar los usos de di (tú) que para reivindicar el papel que esta fórmula cumple en la construcción del discurso, papel que, en general, ha sido ignorado. De hecho, lo normal es que no se la haya incluido entre la nómina de los marcadores del discurso o que sus valores discursivos hayan sido descritos de un modo muy parcial e incompleto.

Los datos en los que se fundamenta nuestro análisis proceden de los corpus de la Real Academia Española (CORDE, CREA y CORPES), del corpus PRESEEA y del corpus telefónico de Olmo Flecha (2005), pero en especial de un corpus de textos, localizados mediante las herramientas de los diferentes buscadores, extraídos de internet ${ }^{3}$. Los criterios que han presidido la búsqueda de los ejemplos de di (tú) que han sido los siguientes: carácter coloquial o que imitan algunas propiedades del registro coloquial, pérdida del significado léxico del verbo decir, pérdida del valor apelativo propio del imperativo y presencia de algún grado de fijación ${ }^{4}$.

\section{EL TRATAMIENTO DE $D I(T U ́)$ QUE EN LA GRAMÁTICA DEL ESPAÑOL}

Pese a ser una fórmula bastante productiva en el ámbito del registro coloquial, apenas ha merecido el interés de los especialistas. Dejando a un lado los estudios de Fernández Bernárdez (2002: 210-213) y Grande Alija (2013), autores que le han prestado cierta

2 Este trabajo se enmarca en el proyecto de investigación titulado "De construcciones periféricas a operadores discursivos: un estudio macro-sintáctico del español" (Ministerio de Economía y Competitividad, FF1201782898-P).

3 El número de ejemplos localizados en los corpus indicados es realmente bajo: un caso en el CORDE, cuatro casos en el CREA, seis casos en el CORPES (uno de ellos también presente en el CREA), tres casos en el PRESEEA (uno de ellos recogido en el CORPES) y dos casos en el corpus telefónico de Olmo Flecha (2005). El corpus elaborado con datos extraídos de internet consta de doscientos treinta ejemplos localizados en diversas búsquedas realizadas a lo largo de enero de 2019. Los ejemplos de internet se reparten según la siguiente tipología de textos: un $53 \%$ corresponden a textos extraídos de redes sociales, un $20{ }^{\prime} 4 \%$ a webs y blogs de diverso contenido, un $17^{\prime} 3 \%$ a textos literarios de carácter coloquial, un $7 \%$ a textos periodísticos y un 2’3\% a conversaciones coloquiales. Los 244 ejemplos identificados en el conjunto de corpus se distribuyen de la siguiente manera: di tú que (195), di que (32), que di (tú) que (17).

4 Para la compilación del corpus se actuó de la siguiente manera: en primer lugar, se procedió a la búsqueda de los casos de di tú que, excluyendo todos aquellos en los que el imperativo mantiene su valor léxico y apelativo; seguidamente, se extrajeron los casos de di que, de nuevo eliminando los ejemplos en que el imperativo sigue manteniendo su valor original. Una vez identificados los tres valores discursivos considerados en este trabajo, se consideró la posibilidad de analizar otras variantes formales. Para ello se estudió el comportamiento de formas como tú di que, vosotros decid que, decid que... y se pudo comprobar que podían descartarse porque no habían sufrido los procesos de transformación semántica y formal detectados en di (tú) que. Por el contrario, se constató la existencia de la variante que di (tú) que (un 7\% de ejemplos). Por último, se entró a considerar el uso de $d i$ tú como estructura parentética. Se pudo establecer que, aunque existía, presentaba unos valores distintos de los identificados para di (tú) que y además circunscritos al español de América. Por estas razones, se decidió dejarlo fuera de nuestro estudio. 
atención, solo se encuentra alguna breve referencia a su papel discursivo en unos pocos diccionarios.

Tal es el caso del $D R A E$, que en su vigésima tercera edición (2014) lo presenta como un procedimiento de intensificación: "expr. coloq. U. a principio de frase para apoyar o encarecer lo que se va a decir. Di que yo estaba cansado, por eso no discuti"'.

Junto al DRAE, se documenta su valor discursivo en el Diccionario fraseológico documentado del español (Seco et alii 2004: 365-366) ${ }^{5}$ y en el Diccionario de partículas de Santos Río (2003). Estas obras tienen el mérito indiscutible de ser de las pocas que han reparado en los valores discursivos de $d i$ (tú) que en el español coloquial. No obstante, presentan un inconveniente: se circunscriben a aspectos muy parciales y no ofrecen una caracterización general de su funcionamiento discursivo.

Por el contrario, no se encuentra la más mínima referencia a los usos discursivos de di (tú) que en trabajos clásicos como los de Beinhauer (1968) y Steel (1976) o en obras tan destacadas como son el Diccionario del uso del español (Moliner 2004), el Diccionario de partículas discursivas del español (Briz et alii coords. 2008) o el Diccionario de conectores y operadores del español (Fuentes Rodríguez 2009). Tampoco aparece recogida en los trabajos de Martín Zorraquino y Portolés (1999) o en el de Landone (2009).

Como ya se ha indicado, un caso aparte es el de Fernández Bernárdez (2002). Apoyándose en Briz (1998), lo sitúa entre los intensificadores pragmáticos que son utilizados "por el locutor para dar mayor fuerza ilocutiva a su acto de enunciación” (Fernández Bernárdez 2002: 193). Otros aspectos destacados de su estudio son los siguientes:

-No recoge la variante di tú que.

-La presenta como "expresión totalmente lexicalizada" (2002: 210).

-Fónicamente, "constituye grupo con el enunciado en el cual incide [...]. Precede siempre a este enunciado" (2002: 210).

-Valor: Con ella el locutor indica que el enunciado que introduce posee mayor fuerza argumentativa (2002: 210).

-Se utiliza tanto en unidades monológicas como dialógicas.

Una última referencia bibliográfica nos la aportan Cortés y Camacho (2005: 197), quienes asignan a di (tú) que un valor de hipótesis.

\section{DI (TÚ) QUE COMO MARCADOR DISCURSIVO}

\subsection{Comportamiento formal de di (tú) que}

Todos los usos discursivos de di (tú) que que nos interesan comparten la propiedad de presentar cierto grado de fijación formal.

La mayoría de los casos recogidos en nuestro corpus presentan la variante di tú que (195 casos, un $80 \%$ del total). Su fijación formal se aprecia en que no es posible variar el pronombre personal tú por otros que tengan referencia de segunda persona ni siquiera para el tratamiento de respeto, ni sustituir el imperativo $d i$ por otras variantes apelativas del verbo decir (decid vosotros que, diga usted que). Tampoco se puede alterar el orden de aparición del imperativo y del pronombre ni es posible insertar otro componente:

5 Esta obra recoge la misma información que Seco et alii (1999). 
(1) En previsión de la más que posible "cogorza" de los clientes, los valdeones se servirán con una tapa de fritos de merluza para acompañar la consumición. «Di tú que para merluza la de Rosa, ¿eh? [...]».

https://www.dariodeleon.es/noticias/72-bar-a6-llamara-valdeones-cachis-honor-rosavaldeon

Una variante documentada, pero con un uso mucho menor, es di que (32 casos, un 13\% del total):

(2) Entonces, lo vais a pagar justos por pecadores. Entiendo que hay gente que es muy honrada, que trabaja el campo, que trabaja el cuero, [etcétera]

XXX.-[La mayoría]

M.T. - pero hay muy mala gente [también]

S.G. $-[$ Todos]

M.T. -y como los conocéis y convivís con ellos, denunciarlos, porque mirar a lo que se dedican, a estafar a la seguridad [social]

S.G.-[Di que] la mayoría son honrados, [porque si no, $\mathrm{xxx}$ ]

(A. Bañón, "Proceso de rectificación en el discurso oral en español")

https://idus.us.es/xmlui/bitstream/handle/11441/39066/2031507_Sintaxis\%201. pdf? sequence $=1$

Otra variante que hemos encontrado es la introducida por que (17 casos, un 7\%):

(3) Esa es una de las cosas más tétricas de las SAD: son totalmente opacas. Los aficionados muchas veces ni siquiera se enteran de lo que pasa. Que di tú que qué más da, si aunque se enteren no pueden hacer nada (https://www.forocoches.com/foro/showthread. php?t=4828849).

$D i$ (tú) que se usa en discursos monológicos y en diálogos. Estos últimos pueden ser tanto conversaciones prototípicas de carácter coloquial como intercambios "conversacionales" realizados a través de medios digitales.

$D i$ (tú) que no puede constituir por sí solo intervención ${ }^{6}$ ni puede aparecer, en los discursos monológicos, como enunciado autónomo. Nunca configura grupo entonativo independiente ni se usa como elemento periférico.

Una de las características generales de $d i$ (tú) que es que siempre -si se dejan fuera ciertos marcadores conversacionales o conjunciones que pueden preceder a esta fórmula ${ }^{7}$ aparece al comienzo de la unidad a la que afecta:

-A comienzo de enunciado (acto):

(4) Buenos y lluviosos días?

Madre mía, la que está cayendo en Madrid, agua y más agua. Di tú que nos viene de maravilla! http://www.iriacastro.com/churros-con-azucar-y-canela-2/

6 Para las unidades conversacionales de intervención, acto y subacto subordinado seguimos la propuesta de Briz y Grupo Val.Es.Co (2003).

7 Hemos documentado los siguientes casos de combinación de di (tú) que con conjunciones y marcadores del discurso: pero (10), y (7), porque (3), pues (3), aunque (1), bueno (7), hombre (3) y total (1). 
-A comienzo de un subacto subordinado:

(5) RE : examenes de jailbreak

Pues en mi opinion tampoco esta tan mal eso de los 3 dias porque di tu que eres nuevo y acabas de hacer el examen y apruebas de chiripa o porque te lo chivan en esos 3 dias $[\ldots]$

http://www.clanzonazero.com/index.php?file=Forum\&page=viewtopic\&forum id $=7 \&$ thread_id $=8296$

-A comienzo de intervención: tanto en una conversación prototípica (el ejemplo 2) como en un discurso dialogal diferido precedido por el marcador bueno:

(6) Bueno di tu que con logica de muñeco de nieve, la lluvia es sangre [Se comenta una viñeta en la que un muñeco de nieve, cuando empieza a nevar, dice: "Pero qué está pasando, ¿está cayendo carne?'].

https://www.cuantarazon.com/993594/que-esta-pasandooooo

-Delante de un sintagma verbal:

(7) En serio granizada de pizza?? puajjjjj, que asco!! [...] Yo soy de probar poco, lo que no me entra por el ojo di tu que no me entra por la boca seguro segurísimo [...]

https://blog.hola.com/hot-shots/2014/10/30-sabores-muy-raros/

-En estructuras parentéticas. $D i$ (tú) que puede integrase en enunciados parentéticos en los que el locutor realiza un comentario al contenido presentado previamente:

(8) En tu biblioteca das al secundario sobre el juego y seleccionas "eliminar contenido local", di tú que no es lo primero que yo haría, antes deberías bajar ciertos aspectos gráficos $[\ldots]$.

https://steamcommunity.com/discussions/forum/29/864976115206375807/

Todos estos contextos de aparición presentan un rasgo en común: di (tú) que no puede iniciar discurso. Presenta una naturaleza netamente reactiva: o bien responde a una intervención previa del interlocutor o bien aparece, en un discurso monológico, desencadenado por lo dicho anteriormente por el propio hablante.

\subsection{Di (tú) que y la aserción}

En la mayoría de los casos di (tú) que introduce una información que el hablante asume como verdadera:

(9) Se olvidan de algo importante. Es amigo de Messi!!! Ustedes creen que dejara de jugar para Argentina y jugar con el mejor del mundo y sus compatriotas? [...]

Para el \#32, ¿amigo de Messi?, di tú que ambos son de Rosario, pero de amiguitos nada $[\ldots]$.

http://www.marca.com/2013/01/27/futbol/futbol_internacional/argentina/1359315852. html 
Aunque la mayoría de los ejemplos se ajustan a este patrón (enunciados declarativos en modo indicativo que apuntan a un hecho aceptado como verdadero), son posibles otros en los que el hablante no presenta el contenido como una realidad cierta e incuestionable.

En una concepción gradual de la aserción, debemos aceptar la posibilidad de que el hablante admita la verdad de un hecho con mayor o menor convencimiento. Se entra así en el terreno de la modalidad epistémica, en la que el hablante no descarta la verdad de algo, pero tampoco la puede admitir sin discusión. $D i$ (tú) que es compatible con estos enunciados modalizados:

(10) Personalmente, el caso que más me ha llamado la atención ha sido el de L'Oreal, por el simple hecho de que Rachel Weisz es una declarada pro-arruga y es un tanto incongruente que ella misma diga no al bisturí, pero sí al photoshop. Di tú que quizás sean decisiones fuera de su alcance.

https://anunciologia.wordpress.com/2012/12/16/y-para-terminar-censura/

En otro orden de cosas, aunque de manera prototípica la aserción suele ir ligada a la presencia de un enunciado declarativo en indicativo, se acepta de modo general que es posible incorporar contenidos asertivos en enunciados que no pertenecen a la modalidad declarativa. $D i$ (tú) que puede aparecer sin problemas en tales entornos enunciativos:

(11) Respecto a la clasificación afirmo rotundamente que todos esos tebeos son basura, aunque no he leído ninguno. Esto es Internet, se pueden hacer este tipo de afirmaciones. La omisión de Scalped es inadmisible. La mejor serie que ha publicado DC en los últimos años y no la incluis en la lista. Dí tú que qué se puede esperar de un atajo de DeCeros.

http://www.zonanegativa.com/dc-comics-lo-mejor-de-2012/

Di que introduce, bajo la forma de una interrogación retórica, un contenido asertivo cuya verdad el hablante acepta de forma plena.

Di (tú) que también aparece con informaciones que se presentan como irreales. Se hace referencia a circunstancias que se sabe que no se han realizado, pero que se podrían haber dado, o bien a situaciones que, aunque no sean reales, no se descarta que lo puedan ser en el futuro:

(12) De casarse me parece genial elegir un vestido, el lugar, la decoración, la comida, o sea amaría planear una boda.

1 respuesta

Todo, menos caminar por un altar. Di tú que lo mío sería ser dama de honor, o alguna vaina de esas. Odio ser el centro de atención. Horror.

https://twitter.com/PrettyNowhere/status/899504081336758272

(13) Hombre di tu que si el cable estuviese "carenado" con un perfil metalico, di tu que seguramente no le hubese pasado nada a Pedrosa [...].

https://www.motorpasionmoto.com/motogp/una-sobre-el-control-de-traccion-y-su-historia

(14) ¿Elección de jueces? No jodas, tendríamos de jueces a rubalcabas, pujoles y rajoys... :P bueno, di tu que al menos los eligirias tu. 
https://www.rankia.com/foros/economia/temas/1645283-propuestas-para-mejorar-nuestro-sistema-democratico

Di (tú) que también aparece asociado a la expresión de contenidos hipotéticos:

(15) Víctima: Ruth Lorenzo (2014).

Objeto sustraido: Secador de pelo.

Consecuencias: La pobre Ruth salió el sábado con el pelo mojado a cantar, y como consecuencia, podía habérsenos resfriado. Di tú que ahora España gana el festival, que por poder, podía haber pasado [...], y ahora se le queda "la voz tomada" que diría mi madre y no puede volver a cantar su Dancing in the rain al final de la gala. Habría sido un drama acuático más grande que Buscando a Nemo.

$\mathrm{http} / / /$ www.sufridoresencasa.com/robos-espana-eurovision/

En él se plantea un mundo alternativo y las consecuencias que se podrían derivar de ese "mundo posible". Aunque no es una manifestación prototípica de la aserción, tampoco está desligada por completo de ella, dado que encara el problema de la verdad en el marco de otro mundo posible distinto del actual.

A modo de recapitulación, podemos sostener que di (tú) que aparece estrechamente unido a la expresión de contenidos asertivos, siempre que mantengamos una visión amplia y gradual de la aserción. En todos estos casos el hablante se enfrenta al problema de la verdad de un contenido proposicional.

No obstante, también puede usarse -aunque de forma muy restrictiva- con enunciados que se adscriben al dominio de la "no aserción" y, en concreto, a la función desiderativa e instrumental del lenguaje:

(16) Me da que Noa y Mario a la larga van a acabar como el rosario de la aurora. Di que para lo que queda de \#CodeBlack, que me aguanten hasta el final, please, que los quiero mucho.

https://twitter.com/BabyCatFace_/status/1015688245546573826

(17) - Di tú que no les hagas caso, Marcial, que ése no es más que un escogido y un propagandista (Sánchez Ferlosio, El Jarama, en Félix Fernández de Castro, Las perífrasis verbales en español actual, Gredos, Madrid, pág. 325).

\subsection{Usos discursivos de di (tú) que}

\subsubsection{Di (tú) que y los fenómenos de intensidad}

Di (tú) que no aporta nada al contenido proposicional del mensaje. La misma representación de la realidad se comunica en un ejemplo como el siguiente, tanto si aparece el marcador en cuestión como si se elimina:

(18) Espérate ahora a su suplencia en La Floja, esa secta culé que viste con la camiseta de España. [...]. Di tú que a mí actualmente me da igual lo que haga ese equipo. Sólo me fastidia que, llevando el nombre de mi país, sea generoso con quienes lo traicionan. http://www.lagalerna.com/isco-ya-vuela/ 
En ambas opciones se está refiriendo a un mismo hecho que se presenta como verdadero. Por tanto, su contribución se orienta hacia el ámbito del significado procedimental, es decir, guía al interlocutor sobre cómo procesar ese contenido proposicional.

Si se compara la versión sin el marcador con aquella en la que sí aparece, se observa que con esta última se refuerza el mensaje. La cuestión que se plantea es determinar en qué consiste este realce expresivo.

A pesar de la estrecha relación que hay entre la noción de verdad y el marcador di (tú) $q u e$, su presencia no supone un incremento del compromiso del hablante con la verdad de lo que dice. Su intensificación no tiene nada que ver con la modalidad epistémica. Di (tú) que puede aparecer con enunciados que representan un hecho seguro, como es el caso del ejemplo anterior, pero también con enunciados modalizados epistémicamente (ejemplo 10: Di tú que quizás sean decisiones fuera de su alcance) que implican un menor compromiso con la verdad de lo que dice. Además, la aparición de di tú que en este último ejemplo no supone el menor cambio, en lo que se refiere a la modalidad epistémica, con relación a la variante sin el marcador. Ambos presentan su contenido proposicional como una mera posibilidad. Finalmente, no hay que olvidar que di (tú) que puede intervenir, aunque en menor medida, en enunciados no asertivos (ejemplos 16 y 17) en los que la cuestión de la verdad no entra en juego.

Para entender la contribución de di (tú) que a la interpretación de este uso, se debe tener presente que su origen se encuentra en una forma apelativa y que, a pesar de los cambios formales y semánticos que ha sufrido, la variante con di (tú) que se orienta de forma clara hacia el interlocutor, busca reforzar el tipo de efecto o repercusión que el hablante espera lograr con el acto ilocutivo. En concreto, aspira a influir abiertamente sobre sus creencias ${ }^{8}$ o, en el caso de los enunciados directivos, sobre su actuación futura. En el ejemplo 18 ( $D i$ tú que a mí actualmente me da igual lo que haga ese equipo), el responsable del mensaje se ve en la necesidad de intensificar el efecto que se espera conseguir en el interlocutor porque es contrario a una expectativa que forma parte del contexto: que aquel es un seguidor de la selección nacional y que, por lo tanto, no le puede dar igual lo que haga ese equipo.

En definitiva, di (tú) que interviene como parte de una estrategia comunicativa de intensificación pragmática ${ }^{9}$ que afecta al conjunto de un acto ilocutivo y con la que se busca conseguir algún tipo de efecto sobre el interlocutor. De acuerdo con una concepción escalar de la intensificación ${ }^{10}$, el enunciado sin el marcador representa el punto de referencia no marcado o neutro respecto al cual di (tú) que intensifica el acto realizado, en especial en lo que se refiere al tipo de efecto que se espera en el interlocutor:

(19) [Contesta a uno que se ha comprado un mac y que está preocupado porque se anuncia el lanzamiento de otro nuevo]

8 En los usos intensificadores de una aserción, di (tú) que es fácilmente intercambiable por el marcador parentético créeme (Grande Alija 2014).

9 La intensificación pragmática se entiende como una estrategia comunicativa, centrada en el contenido proposicional o en la modalidad, y con la que el hablante pretende producir ciertos efectos en el nivel comunicativo y social (Albelda Marco 2007: 166). Véase Meyer-Hermann (1988), Bazzanella et alii (1991), Caffi (1999), Sbisà (2001), Briz (1996, 1998), Albelda Marco (2007), entre otros.

10 Según Albelda Marco (2007: 112), “[1]a noción semántica común a las formas de intensificación es la de escala”. Una escala está configurada por un conjunto ordenado de elementos, que poseen un rasgo común en diferentes grados, lo cual permite hablar de miembros más fuertes y miembros más débiles (2007: 118). 
No te preocupes... pasan bien los años por ellos; no obstante, si la modernización del modelo fuese quitarle puertos, dí tú que te vas a reír de los que compren el nuevo. https://www.applesfera.com/rumores/iphone-6ss-7-7s-7-pro-en-que-quedamos-rumorsfera

(20) Al lumbago se añade la amiga ciática, que es cuando de repente te da un pinchazo en el culo que te recorre toda la pierna hasta el tobillo y di tú que de ahí ya ni te mueve ni el tato.

http://laaventurademiembarazo.com/2015/06/mis-24-y-25-semanas-de-embarazo.html

A modo de resumen, las principales características de di (tú) que como intensificador pragmático son las siguientes:

- Di (tú) que, en virtud de la orientación intersubjetiva que se deriva de su origen apelativo, busca reforzar el efecto que el hablante pretende conseguir en su interlocutor.

-Se intensifica, con relación a una variante más neutra, un acto de habla.

-Proporciona énfasis a la información. Este realce puede ser aprovechado con fines argumentativos.

-Puede confluir parcialmente con otros marcadores (por ejemplo, la verdad) que también contribuyen a destacar cierta información y a reforzarla argumentativamente.

Junto a su función intensificadora encontramos casos en los que se usa con una evidente función atenuadora:

(21) ¿Qué quiere de comer la niña?

Lo que haya para todos, ¿o acaso a mí me alimentaban a la carta?. Dí tú que había platos que me gustaban más y yo sé, aunque ella no lo dijese, que los hacía con más frecuencia para satisfacerme. Pero de ahí a que haya que preparar un menú especial para las nenas hay un trecho.

https://mujeresymadresmagazine.com/padres-ayer-abuelos-hoy/

(22) En tu biblioteca das al secundario sobre el juego y seleccionas "eliminar contenido local", di tú que no es lo primero que yo haría, antes deberías bajar ciertos aspectos gráficos $[\ldots]$.

https://steamcommunity.com/discussions/forum/29/864976115206375807/

Comentamos a continuación brevemente estos ejemplos para mostrar cómo actúa este tipo de estrategia.

En el ejemplo 21 aparece una interrogación retórica (¿acaso a mí me alimentaban a la carta?) que conduce a la interpretación de que "a mí no me alimentaban a la carta", entendida en el sentido de que su madre no le daba de comer todo lo que le apetecía en cada momento. Sin embargo, en el siguiente enunciado di (tú) que introduce una información que relativiza la contundencia de esa información.

En el ejemplo 22 el locutor da un consejo a su destinatario: En tu biblioteca das al secundario sobre el juego y seleccionas "eliminar contenido local". Sin embargo, la validez del consejo queda puesta en entredicho de inmediato al admitir, mediante un enunciado parentético introducido por di (tú) que, que eso no sería lo primero que haría él.

Con relación al valor de atenuación de di (tú) que, resulta oportuno hacer las siguientes precisiones: 
-Pensamos que el valor de di (tú) que es fundamentalmente ser un procedimiento de intensificación de un acto de habla. No obstante, no se debe descartar que pueda aparecer asociado a algún tipo de estrategia de atenuación discursiva.

-La cuestión que de inmediato se plantea es la siguiente: ¿cómo es posible que un procedimiento destinado aparentemente a intensificar un acto de habla acabe participando en una estrategia de atenuación?

-Esto es posible porque en estos casos di (tú) que intensifica una ilocución cuyo contenido desarma, desactiva o debilita los supuestos pragmáticos ligados a un enunciado previo.

De algún modo, en esta clase de ejemplos se intensifica para atenuar. En realidad, este tipo de interferencia no es tan extraña porque intensificación y atenuación no son más que dos orientaciones (ascendente, hacia valores de más fuerza / descendente, hacia valores de menos fuerza) de un mismo fenómeno de intensidad que se define como "la expresión de diferentes valores relativos en una escala, que aumentan o disminuyen respecto a un punto de referencia" (Mihatsch y Albelda Marco 2016: 8). Estas mismas autoras ponen de relieve que aunque ambos fenómenos se ven como contrarios al ir en direcciones opuestas, "los usos reales de la lengua ofrecen casos de polifuncionalidad intensificadora y atenuadora en una misma expresión, bien en usos distintos o bien en los mismos usos contextuales" (2016: 11). Por tanto, el caso de di (tú) que nos sería muy distinto del de otros procedimientos.

\subsubsection{Intensificación en un contexto de antiorientación argumentativa}

En aproximadamente un $41 \%$ de casos los ejemplos de nuestro corpus aparecen integrados en entornos de contraargumentación en los que representan el miembro de más fuerza argumentativa. A decir verdad, todo parece preparado para que este marcador acabe señalando un cambio en la orientación argumentativa del discurso que se está desarrollando. Dado que este marcador muestra una clara tendencia a especializarse en intensificar un acto asertivo, lo normal es que su contenido proposicional represente, en un proceso de contraargumentación, el argumento que conduce a la conclusión que prevalece en la continuación del discurso. La presencia de conectores de naturaleza contraargumentativa no hace sino reforzar este comportamiento:

(23) ¿Y cómo funcionan tus tarifas?

[...] La tarifa varía mucho dependiendo de la complejidad del tema y del cliente. Pero di tú que un ensayo que toma un día de trabajo le cuesta a un cliente entre $60.000 \mathrm{y}$ 80.000 pesos.

https://www.vice.com/es_co/article/4w9bxj/esta-es-la-gente-que-se-ofrece-a-hacer-sustrabajos-de-la-universidad-por-plata

Con todo, lo más frecuente es que no se incluya ningún tipo de marcador contraargumentativo, y que el valor de antiorientación se resuelva pragmáticamente como consecuencia de contraponer en el discurso dos proposiciones que, argumentativamente, van en direcciones opuestas. La introducida por $d i$ (tú) que conduce a la conclusión de más peso, tal como ocurre en este ejemplo: 
(24) Buenos y lluviosos días?

Madre mía, la que está cayendo en Madrid, agua y más agua. Di tú que nos viene de maravilla!

http://www.iriacastro.com/churros-con-azucar-y-canela-2/

El comentario inicial sobre la gran cantidad de lluvia que está cayendo en Madrid parece apoyar la conclusión de que se trata de algo molesto, no deseado. El enunciado que introduce di tú que desactiva claramente esta conclusión.

A pesar de que di (tú) que aparece con frecuencia integrado en una estructura contraargumentativa en la que representa el argumento más fuerte, ello no significa que siempre sea así. De hecho, la intensificación que expresa no es incompatible con la posibilidad de que pueda aparecer introduciendo el componente más débil dentro de una conexión entre argumentos antiorientados, tal como se puede apreciar en el ejemplo 9 (di tú que ambos son de Rosario, pero de amiguitos nada).

\subsubsection{Di (tú) que como inductor de ironía}

En este nuevo valor también se aprecia intensificación y referencia a un contenido que se asume como verdadero, pero con la particularidad de que se promueve una interpretación irónica con cambio de polaridad:

(25) - SENÉN.- Yo no sé sino que el viejo Albrit, que hasta ahora, desde la muerte de su hijo, no se ha movido de Valencia, escribió a la Condesa...

- VENANCIO.- (Riendo.) Pidiéndole dinero.

- SENÉN.- Hombre, no: le proponía una entrevista para tratar de asuntos graves...

- GREGORIA.- De asuntos de familia. Y como la Condesa no quiere altercados en Madrid, porque allí puede haber escándalo, [...], le ha citado en este rincón de Jerusa, donde sólo vivimos cuatro papanatas, y si hay zipizape aquí se queda, y la ropa sucia en casita se lava. ¿Qué tal, señor cortesano, entiendo yo a mi gente?

- VENANCIO.- Di que no es lista mi mujer.

- SENÉN.- (Risueño y galante.) Sabe griego y latín. ¡Vaya un talento! [...]

(B. Pérez Galdós, El abuelo, 1897, Biblioteca Virtual Miguel de Cervantes).

(26) - ¿Hace mucho que viniste?

-Serían las dos y cuarto.

- ¡Pues di tú que no te gusta tomar sol desde temprano!

-Es que si no te adelantas, se te ponen unos cuantos golfos en primera fila y no ves el espectáculo (Félix Limendoux, "El tendido de los sastres", Blanco y Negro, 10/11/1900, pág. 18).

http://hemeroteca.abc.es/nav/Navigate.exe/hemeroteca/madrid/ blanco.y.negro/1900/11/10/018.html

Por su interpretación podemos adscribir esta clase de ejemplos al ámbito de la exclamación pragmática. En ellos se refuerza expresivamente una proposición que se asume como verdadera, tal refuerzo viene determinado por lo que parece una intensificación de una propiedad gradual o de una cantidad. Así, en el ejemplo 25, el locutor no está dando a entender que su mujer es lista, sino más bien que es "muy lista". 
Se rechaza una proposición para implicar que se asume la contraria. Aunque parece que el locutor está apoyando cierta proposición (di tú que no p p), en realidad pragmáticamente está defendiendo la contraria $(p)$. En otras palabras, di (tú) que promueve una interpretación en antífrasis con cambio de polaridad.

Teniendo en cuenta esto, podemos afirmar que di (tú) que mantiene el valor básico de intensificación del contenido de un enunciado de naturaleza asertiva y que el cambio de polaridad que se produce en esta clase de ejemplos hay que analizarlo a la luz del funcionamiento general de los enunciados irónicos: la información lingüística entra en contradicción con un contexto comunicativo en el que prevalece la opción contraria, que es por la que se decanta el hablante:

-Información lingüística: di (tú) que no $p$

-Información contextual: $p$, por tanto, prevalece $p$

Pensamos que la presencia de la negación en la antigua oración sustantiva es determinante para entender el funcionamiento de estas estructuras. La oración negativa anticipa un posible rechazo por parte del interlocutor de un contenido asertivo, pero este rechazo se contrapone a una evidencia aportada contextualmente en sentido contrario y, a los ojos del hablante, más fuerte que la que pueda apoyar el punto de vista opuesto. Hay, por tanto, un inequívoco sentido polémico. Algo parecido ocurre en este otro ejemplo:

(27) «No puedes figurarte - decía a su marido, al salir de un taller-, cuánta lástima me dan esas infelices muchachas [...]. No tienen educación, son como máquinas, y se vuelven $\tan$ tontas... [...] que en cuanto se les presenta un pillo cualquiera se dejan seducir $[\ldots]$ ». -Filosófica está mi mujercita.

-Vaya... di que no me he lucido... [...](B. Pérez Galdós, Fortunata y Jacinta, 1887, Biblioteca Virtual Miguel de Cervantes).

Este ejemplo presenta además la particularidad de que está en primera persona, es decir, que el juicio expresivo se refiere a la propia persona que lo hace. Este hecho tiene su importancia, dado que parece favorecer un sentido de reproche o desafío, lo que hace viable una paráfrasis como "¡atrévete a decir que no me he lucido!”, que sugiere cierta pervivencia de la función interactiva de la modalidad apelativa.

Para finalizar el análisis de este valor nos parece interesante un ejemplo que muestra un paso más en la evolución de di (tú) que en estos contextos irónicos. En él di tú que no aparece como un elemento marginal que se usa parentéticamente tras un enunciado asertivo en el que se afirma el contenido proposicional que el hablante acepta como verdadero:

(28) Que Japón esté en octavos supone una extravagancia de improbable justificación

En respuesta a@jg_dominguez

es una extravagancia encantadora, di tu que no!! ... además, todos con JAPON!!

https://twitter.com/Munttse/status/1013851094701756416

\subsubsection{Di (tú) que con valor de hipótesis}

Otro valor de di tú que que se encuentra bien representado en nuestro corpus es el de hipótesis. De los diversos autores que se han ocupado del análisis de esta fórmula solo 
Cortés y Camacho (2005) han reparado en él. A los ejemplos 5 y 15 podemos añadir los siguientes:

(29) «... sí, pero ahora di tú que tienes el sida, ¿tú le vas a decir a la nena con la que vas a salir...? [...] Pero eso es, yo no le diría a una nena que tengo el sida, la verdad. [...]» (J. L. Bimbela y G. Maroto, Mi chico no quiere usar condón)

file://C:/Users/MICROSOFT/Downloads/EASP_Mi_chico_no_quiere_usar_condon. pdf

(30) Iniciadores de conversación (por ejemplo: ¿qué te gusta hacer en una primera cita?)

Algo breve, di tu que no te guste.....

https://www.pof.com/viewprofile.aspx?profile_id=126530681

(31) ¿Se te ocurre alguna idea? Si se te ocurre dímela que cuanto más loca más capaz soy de llevarla a cabo... bueno, bueno eso es una fanfarronada, no me creas mucho que yo soy muy cobardica... pero tú propón... di tú que me diera por ahí.

http://www.trebolarium.com/lectores/nutricion/piensa-y-adelgaza/la-chica-del-guardaespaldas-no-encontro-la-salida/

(32) Yo puedo comprender que sea un coñazo, incómodo, bla bla bla. [...]. Pero querer quitarse la barba para siempre... Qué mal rollo! Di tú que dentro de dos semanas quieres dejarte perilla o lo que sea.

https:/vandal.elespanol.com/foro/mensaje/424829/depilacion-laser-zona-barba-postpara-gente-que-se-la-haya-hecho-a-ser-posible/

(33) [...] mi madre se cayó de unas escaleras, entonces fue al médico y lo primero que hicieron fue apartar a mi padre para preguntarle si el moratón que tenía en la cara había sido, que se lo había hecho... y lo hablamos con total naturalidad y nos parecía... Fue un momento cómico y positivo porque di tú que hubiera sido verdad que mi madre a lo mejor... (I. Postigo y A. Jorge (coords.), El tratamiento informativo de la violencia contra las mujeres, DOI: $10.4185 / \mathrm{cac} 86)$.

Los ejemplos anteriores tienen en común la propiedad de que expresan una hipótesis, es decir, una suposición que, de llevarse a cabo, podría implicar o haber implicado ciertas consecuencias. En ellos, di (tú) que se comporta como una fórmula plenamente fijada e idiomática y se podría sustituir por otras fórmulas apelativas (imagina, supón que, pongamos que...) que cumplirían la misma función. Aunque el significado apelativo de decir se ha perdido por completo, es indiscutible que está presente en esta fórmula una evidente orientación apelativa o intersubjetiva, puesto que se trata de animar al interlocutor a que considere lo que podría suceder en otro mundo alternativo. En la mayoría de los ejemplos recogidos la implicación pragmática que se derivaría de la suposición planteada queda implícita.

Mientras que en el caso de los usos analizados en los apartados anteriores, se siguen manteniendo los mismos actos de habla básicos aun cuando no aparezca introduciéndolos la fórmula $d i$ (tú) que, en el caso de este uso hipotético su presencia es ineludible para alcanzar este valor, puesto que su ausencia o bien conduce a una aserción plena sin el más mínimo sentido hipotético (Di tú que ahora España gana el festival > ahora España gana el festival) o bien el resultado es inequívocamente agramatical (Di tú que no te guste $>* N o$ 
te guste). Significa esto que di (tú) que en este tipo de uso no puede entenderse como un elemento que modifica un acto de habla plenamente autónomo, al que refuerza o intensifica, sino que actúa como un componente imprescindible para que el hablante pueda realizar el acto de plantear una hipótesis.

Según Cortés y Camacho (2005: 196), los marcadores de relación jerárquica de tipo argumentativo, entre los que se incluye $d i$ (tú) que, "tienen en cuenta tanto el acto o unidad que precede al marcador como el que le sigue, por su vinculación semántica y pragmática; por esta causa, no se pueden desligar para la intelección del enunciado que constituyen". En otras palabras, di (tú) que implica la presencia de dos componentes o dos actos que se exigen mutuamente: uno -el introducido por $d i$ (tú) que- actúa como marco hipotético del que se deriva la consecuencia expresada por un segundo acto. Se trata de relaciones discursivas y pragmáticas entre actos o enunciados, no de relaciones sintácticas. Esto explica que con gran frecuencia el segundo miembro no aparezca explícito, bien porque no es necesario al ser fácilmente deducible del contexto, bien porque no se quiere o no se puede concretar. En todo caso, hablamos de relaciones entre enunciados o actos. El segundo componente puede ser, como ocurre en 29, un enunciado interrogativo (pero ahora di tú que tienes el sida, ¿tú le vas a decir a la nena con la que vas a salir...? A su vez, la unidad discursiva que conforman puede a veces intervenir todo ella como justificación de otro acto de habla, tanto si aparece introducido por una conjunción explicativa: (33) Fue un momento cómico y positivo porque di tú que hubiera sido verdad que mi madre a lo mejor...) como si no: (30) Algo breve, di tu que no te guste

Di (tú) que con valor hipotético introduce siempre una estructura oracional. Llama la atención la diversidad modal y temporal del núcleo verbal de esta oración, diversidad que hace posible que el sentido hipotético adquiera varios matices. Puede aparecer tanto en indicativo como en subjuntivo. En algunos de los ejemplos recogidos la hipótesis que se realiza se presenta como contrafactual, es decir, hace referencia a un hecho que aunque pudo ser cierto, finalmente no lo fue. Con este valor encontramos el uso del pretérito pluscuamperfecto de subjuntivo como ocurre en el ejemplo 33, pero el presente de indicativo puede asumir la misma función, como se aprecia en 15 (Di tú que ahora España gana el festival). En el resto de casos esa hipótesis se plantea como posible, es decir, como no descartable en un futuro más o menos inmediato. La posibilidad del cumplimiento de esa hipótesis puede verse como más factible (con presente de indicativo o subjuntivo) o como de más difícil realización (con pretérito imperfecto de subjuntivo: di tú que me diera por ahi).

Antes hemos destacado que $d i$ (tú) que con valor hipotético podría sustituirse por otras fórmulas apelativas como supón que, imagina que, pongamos que... Queremos ahora fijarnos brevemente en la última de ellas porque, aparte de estar ya en un proceso claro de gramaticalización, nos puede ayudar a comprender mejor el uso de di (tú) que.

Según C. Fuentes Rodríguez, pongamos (que), al que clasifica como operador enunciativo, "[p]recede e introduce una situación hipotética que va a usarse como ejemploargumento" (2009: 257). En la misma obra (2009: 258) se refiere a Pongamos 2, operador enunciativo que "se intercala en el enunciado e introduce un elemento que presenta con un valor ejemplificador y a la vez hipotético. Es una situación posible que se emplea en su valor paradigmático".

Se observa que existe una estrecha relación entre la expresión de una hipótesis y el valor ejemplificador. Se trata de una estrategia plenamente coherente dado que, al mismo tiempo 
que se concibe una situación hipotética, se la presenta como ejemplo concreto, entre otros muchos posibles, sobre el que fundamentar una argumentación.

En el caso de di (tú) que, el valor hipotético de los ejemplos recogidos en este apartado está fuera de toda duda. No obstante, en la mayoría de ellos no hay ningún problema para que aparezca acompañado de un marcador como por ejemplo. Esto no sería más que la consecuencia esperable de plantear, entre otras muchas posibles, una situación hipotética que, de este modo, acaba presentándose como paradigmática. En el caso del español peninsular no hemos encontrado ejemplos del uso de $d i$ (tú) que con un valor puramente ejemplificador. Sin embargo, es una potencialidad que de algún modo está latente tras el valor hipotético descrito. De hecho, en el español de América hemos documentado ampliamente un uso di tú, ya sin la conjunción, siempre en construcción parentética y antepuesto a un elemento nominal, con el inequívoco valor de por ejemplo. Sería un paso más en el proceso de gramaticalización de una fórmula usada inicialmente para expresar una hipótesis:

(34) Ni al caso esta comparación. Uribe solo puede ser comparado con, di tú, Pablo Escobar. 8:33 - 30 jul. 2018 https://twitter.com/viddes_/status/1023954632022941697

(35) "Jaguares siempre ha sido de la casa. Con la institución nos une un vínculo afectivo y deportivo de vieja data así que esto es más como, di tú, presentarle la novia a los papás $[\ldots] "$.

https://actualidadpanamericana.com/jaguares-anuncio-dimayor-patrocinador-2018/

Para terminar, quisiéramos llamar la atención sobre el hecho de que a pesar de que estos usos hipotéticos parecen semánticamente y formalmente bien delimitados, no se pueden desligar por completo de los otros valores de di (tú) que. En algunos casos, ciertas propiedades formales favorecen claramente el valor hipotético (presencia de formas verbales de subjuntivo), pero en otros casos (como cuando aparecen formas de indicativo, especialmente de presente de indicativo) el sentido hipotético solo se puede establecer contextualmente: di tú que está lloviendo, por lo que no puedo salir [valor asertivo] / $\underline{d i}$ tú que está lloviendo y no tienes donde resguardarte, ¿qué harías? [valor hipotético]. Solo considerando cómo continúa el discurso en cuestión se puede determinar si se está refiriendo a un hecho real o si, por el contrario, se trata de una simple hipótesis. Asimismo, en los dos casos di (tú) que trata de focalizar la atención del interlocutor sobre un contenido que, bien se conciba como real o bien como puramente hipotético, queda realzado: ya sea para reaccionar a lo que se acaba de decir, ya sea para seleccionarlo entre otras muchas alternativas posibles que también se podrían haber considerado, pero que finalmente han sido obviadas por no ser pertinentes.

\section{DE CONSTRUCCIÓN VERBAL A MARCADOR DEL DISCURSO}

\subsection{Modalidad apelativa y marcadores del discurso}

Es indudable que la construcción verbal de la que ha surgido el marcador di (tú) que contaba con al menos dos rasgos que han ayudado a desencadenar esa evolución. Nos referimos, en primer lugar, al valor apelativo que presenta por incluir un verbo en modo impe- 
rativo y, en segundo lugar, a la presencia de un significado léxico como decir que designa la realización de un acto de enunciación.

Estos dos aspectos están presentes en muchos marcadores del discurso. Por ejemplo, la orientación apelativa se aprecia, al menos en su origen, en todos aquellos construidos sobre la base de la modalidad interrogativa ¿entiendes?, ¿sabes?, ¿verdad?, ¿estamos?... o sobre formas verbales de imperativo o subjuntivo apelativo. La mirada interna al propio acto de enunciación es la que hace que numerosos marcadores se hayan constituido a partir del verbo decir.

El llamado modo imperativo ocupa en nuestra lengua una posición muy especial porque se puede caracterizar al mismo tiempo como modo y modalidad. Al integrarse morfológicamente en el paradigma verbal se opone a otros modos como el indicativo o el subjuntivo, pero por su contribución al significado del enunciado se comporta como una modalidad de enunciación distinta, por ejemplo, de la declarativa o de la interrogativa.

Teniendo en cuenta lo dicho, es evidente que el origen de di (tú) que no está solo en una forma verbal en imperativo, sino en lo que puede caracterizarse como la unión de una estructura sintáctica (núcleo verbal + sujeto + oración completiva) asociada a la presencia de una modalidad de enunciación apelativa, es decir, un esquema enunciativo (Grande Alija 2016). La pregunta que surge de inmediato es la siguiente: ¿Qué queda de todo ese conglomerado sintáctico-semántico, de ese patrón enunciativo que está en el nacimiento de di (tú) que como marcador discursivo? La respuesta que se dé nos dirá el punto en que se encuentra su evolución.

Puede ser interesante considerar este proceso de pérdida del significado léxico y gramatical, de transformaciones formales que sufre en su evolución hacia lo que es un marcador del discurso, no de forma aislada, sino a la luz del comportamiento que muestran otros usos del imperativo $d i$ en el ámbito del discurso, así como otras formas verbales apelativas que ya actúan claramente como marcadores.

Con relación a los primeros, en Grande Alija (2013) defendimos que las diversas fórmulas discursivas en las que aparece el imperativo $d i$ podían explicarse como puntos destacados en una escala en la que se tuviera en cuenta el cambio gradual que afectara principalmente a los siguientes parámetros:

-En el plano formal: grado en que se mantienen las mismas propiedades formales (sintácticas y morfológicas) de la combinatoria sintagmática original.

-En el plano semántico:

-Grado en que pervive la modalidad apelativa y, en contrapartida, grado en que surgen nuevos contenidos modales y discursivos;

-grado en que el significado léxico del verbo decir está todavía presente;

-y, en consecuencia, grado en que el significado de la combinatoria sintagmática es

el resultado de la suma de los significados de sus componentes.

Estas propiedades se pueden explicar en términos de fijación formal e idiomaticidad ${ }^{11}$, de modo que hay una correlación clara entre ellas y la transformación de lo que era una forma verbal apelativa (más ciertos complementos) en otra categoría.

11 Estas dos propiedades nos adentran en el campo de las unidades fraseológicas (Zuluaga 1980, Corpas 1996, Ruiz Gurillo 1997). La primera se define como "complejidad o estabilidad de forma y, adicionalmente, como defectividad combinatoria y sintáctica" (Ruiz Gurillo 1998: 12). Que un sintagma esté fijado supone que "siempre se reproduce del mismo modo y que no admite grandes variaciones en su estructura" (Ruiz Gurillo 2001: 19). La otra propiedad consiste en que "el significado de la estructura no puede deducirse del significado de sus partes, tomada por separado o en conjunto" (Ruiz Gurillo 1998: 12). 
El siguiente esquema intenta reflejar ese recorrido escalar:

$(+)$ modalidad apelativa

(-) fijación

(-) idiomaticidad

(-) GRAMATICALIZACIÓN
(-) modalidad apelativa

(+) fijación

$(+)$ idiomaticidad

(+) GRAMATICALIZACIÓN

De izquierda a derecha, encontraríamos los siguientes usos discursivos del imperativo $d i^{12}$ : en primer lugar, aquellos en los que el significado apelativo funciona a pleno rendimiento, pero en los que ya se aprecian ciertas restricciones en la combinatoria sintagmática. En segundo lugar, aparecen otras realizaciones en las que, a pesar de que el valor imperativo está todavía de alguna forma presente, su funcionamiento en el discurso se distancia claramente de aquel significado primario. Este cambio en su función comunicativa va unido a una inequívoca fijación formal. Finalmente, se tienen realizaciones en las que a la ausencia de significado apelativo, consecuencia de su carácter idiomático, se añade una fuerte fijación formal.

Respecto a otros marcadores discursivos con origen en formas verbales apelativas, nos interesan los que se han desarrollado a partir de verbos de percepción. Sus propiedades más destacadas serían las siguientes:

(i) tienen un carácter parentético [...]; (ii) son formas imperativas o relacionadas con el imperativo; (iii) tienen un valor conativo básico que las vincula con el receptor, comunicativamente, y, por ende, a la segunda persona (tú o usted), por lo que no son completamente invariables desde el punto de vista morfológico y conservan la flexión para la segunda persona de cortesía; (iv) tienen valores relacionados con la gestión de la interacción, por lo que (v) son formas típicas de la lengua oral (Guillén Escamilla 2018: 132).

Formas como oye-oiga, mira-mire, escucha-escuche, fijate-fijese han sufrido un proceso de gramaticalización que ha acabado por transformarlos en marcadores del discurso. Dejan de actuar como verbos plenos en torno a los que se incardinan diversos tipos de complementos, pierden capacidad de variar los morfemas flexivos (solo admiten cambios relacionados con el tratamiento de respeto), suelen quedar al margen de la curva melódica del enunciado y pueden sufrir algún tipo de reducción fónica. Lo curioso de todo esto es que, precisamente como consecuencia de su origen en formas de imperativo, y a pesar de los cambios sufridos, mantienen cierto valor de naturaleza apelativa porque cumplen una función intersubjetiva orientada hacia el receptor.

Si comparamos el grado de evolución de di (tú) que con el comportamiento de algunos de los marcadores discursivos que tienen su origen en otras formas verbales apelativas, encontramos puntos de confluencia, pero también diferencias:

a) Pérdida o mantenimiento del significado léxico del verbo en imperativo. La pérdida es clara en el caso del valor intensificador y del valor hipotético. El comporta-

12 Para los detalles del comportamiento de cada fórmula del imperativo di remitimos a Grande Alija (2013). 
miento de estos dos usos se aparta del de los marcadores en los que sí hay cierta pervivencia del significado original de la forma verbal apelativa (oye, fijate, escucha...). Como consecuencia de este comportamiento, no hay composicionalidad: el significado de la fórmula discursiva no es resultado de la suma del significado de sus componentes. Presenta un carácter idiomático. El caso de los usos irónicos es distinto. Su valor discursivo consiste en realzar un contenido que el hablante asume como incuestionable. Sin embargo, de algún modo el significado de $d i$ está todavía de alguna forma presente y es fácil reconstruir la estrategia que lleva desde el valor apelativo al sentido final: el hablante desafía al oyente a que se atreva a decir algo que en ese contexto es obviamente falso, de ahí el cambio de polaridad que se produce en la interpretación final. Por lo tanto, di (tú) que como inductor de ironía se encuentra un paso por detrás en la evolución hacia un componente plenamente gramaticalizado.

b) Fosilización de los morfemas flexivos: no admite ningún tipo de variación en estos morfemas, ni siquiera en los relacionados con la segunda persona y las formas de tratamiento, que suelen ser los más resistentes. Los marcadores con verbos de percepción de valor apelativo sí admiten todavía este tipo de transformación. En el caso del valor irónico de di (tú) que, y teniendo en cuenta lo dicho en a), no es descartable que se puedan hacer alguna transformación en los morfemas flexivos relacionada con las formas de tratamiento y el plural. Nosotros no hemos detectado ningún ejemplo, al menos claro.

c) Fosilización sintáctica. Como consecuencia de la pérdida de su condición de verbo, el imperativo di ya no subcategoriza ningún tipo de complemento: tú ya no es el sujeto de esa forma verbal, ni la estructura que sigue a la conjunción que actúa como complemento directo suyo (no hay posibilidad de sustituirla por un referente pronominal neutro). De nuevo, la nota discordante parece ponerla el valor irónico. En algunos ejemplos de este tipo la estructura completiva podría ser sustituida por el pronombre neutro de complemento directo: Vaya... di que no me he lucido (ejemplo 27), podría transformarse en Vaya... di que no me he lucido. Venga, dilo. Sin embargo, en este tipo de ejemplo el sentido de desafío y reproche del imperativo está todavía muy vivo $\mathrm{y}$, por ese motivo, no es una muestra prototípica de esta interpretación.

d) Pérdida de modalidad apelativa: mientras que en el caso de los marcadores construidos a partir de verbos apelativos de percepción que, dada su naturaleza interactiva orientada hacia el interlocutor, mantienen todavía cierta conexión con la modalidad apelativa, en el de di (tú) que la situación es más compleja y depende de los valores discursivos que estén en juego. La pervivencia del valor modal apelativo está íntimamente unida a la del significado léxico del verbo decir. De nuevo aquí se ha de hacer una diferencia entre el comportamiento, de un lado, de los usos de di (tú) que con valor intensificador y di (tú) que con valor hipotético y, del otro, de di (tú) que con valor irónico. En el caso de los dos primeros, a la desaparición del significado léxico de decir se le une la del valor modal apelativo del verbo en cuestión. En el uso de di (tú) que como inductor de ironía hay una pervivencia, aunque sea solo en un plano subyacente, de las dos dimensiones significativas. A primera vista su valor final parece 
tener poco que ver con la modalidad apelativa, pues se trata de realzar la verdad de un contenido proposicional que es abiertamente contrario a lo que se afirma de manera explícita. Sin embargo, se puede reconstruir el proceso deductivo que nos lleva desde la modalidad apelativa hasta el valor irónico: di tu que no $\boldsymbol{p}$, [contexto: $p$ ], luego $i p$ !. No se trata de un cambio de significado, sino más bien de la consolidación de una rutina discursiva, tal como ocurre en el caso de ¿Puedes abrir la ventana?, cuyo significado no es ser una petición, aunque, por una convención de uso (Morgan 1978), lo esperable, lo normal es que se entienda como una petición y no como una simple pregunta. En realidad, pese a los evidentes cambios semánticos sufridos en los usos intensificadores e hipotéticos, se debe reconocer que los dos siguen manteniendo una cierta orientación hacia el oyente, especialmente los usos hipotéticos. De hecho, como se ha visto, el $d i$ (tú) que hipotético es fácilmente sustituible por otras formas verbales apelativas como supón que, imagina que... Esta orientación intersubjetiva no deja de estar presente incluso en el caso de los valores intensificadores: con di (tú) que el hablante intenta focalizar la atención del interlocutor en una información y convencerle de la verdad de la misma.

e) Construcción parentética. Finalmente, y a diferencia del comportamiento de otros muchos marcadores con origen en la modalidad apelativa, di (tú) que no puede aparecer en construcción parentética. El motivo para esta incapacidad se encuentra en la presencia de la conjunción que. No obstante, es necesario hacer alguna matización. En el caso del uso como inductor de ironía, en lugar de encontrar la estructura esperable "di tu que no p", hemos documentado un ejemplo (28) con esta variante: " $p$, di tú que no", en el que el marcador aparece negado en el margen derecho como comentario parentético que afecta a un contenido proposicional afirmativo. Finalmente, hemos localizado en el español de América, ciertos usos parentéticos de di tú con un claro valor ejemplificador que no hacen más que consolidar un sentido que, como ya se ha destacado, estaba de alguna forma latente en el valor hipotético de di (tú) que.

En definitiva, la evolución desde una construcción verbal que funciona como esquema enunciativo de modalidad apelativa a lo que ya es otro tipo de categoría que interviene en el procesamiento de unidades del discurso es un paso gradual. Los tres valores que hemos considerado aquí de $d i$ (tú) que configuran una escala en la que el valor intensificador y el valor hipotético se sitúan en la parte de la derecha (mayor gramaticalización, mayor fijación, mayor idiomaticidad), mientras que el valor como inductor de ironía ocupa una zona intermedia (menor gramaticalización, menor fijación, menor idiomaticidad).

\subsection{Di (tú) que y su categorización como marcador del discurso}

En la siguiente tabla se recogen aquellas propiedades que con frecuencia suelen aducirse (véase Landone 2009) a la hora de caracterizar una unidad como marcador del discurso: 


\begin{tabular}{|c|c|c|c|}
\hline & $\begin{array}{l}\text { Di tú que de } \\
\text { intensificación }\end{array}$ & $\begin{array}{l}\text { Di tú que como } \\
\text { inductor de } \\
\text { ironía }\end{array}$ & $\begin{array}{l}\text { Di tú que } \\
\text { hipotético }\end{array}$ \\
\hline $\begin{array}{l}\text { Función asociada al discurso en } \\
\text { alguno de sus planos }\end{array}$ & Sí & Sí & Sí \\
\hline Independencia fónica y entonativa & No & No & No \\
\hline Carácter extrapredicativo & No & No & No \\
\hline $\begin{array}{l}\text { Conexión (semántica, pragmática, } \\
\text { contextual, textual) }\end{array}$ & Sí & Sí & Sí \\
\hline Pérdida del significado léxico & Sí & Sí-no & Sí \\
\hline $\begin{array}{l}\text { Modificaciones semánticas } \\
\text { (idiomaticidad) }\end{array}$ & Sí & Sí-no & Sí \\
\hline $\begin{array}{l}\text { Al margen del significado } \\
\text { proposicional }\end{array}$ & Sí & Sí & Sí \\
\hline Fijación formal & Sí & Sí-no & Sí \\
\hline Movilidad & No & No & No \\
\hline $\begin{array}{l}\text { Combinación con conjunciones } \\
\text { copulativas }\end{array}$ & Sí & Sí & Sí \\
\hline Autonomía & No & No & No \\
\hline $\begin{array}{l}\text { Está asociado convencionalmente a } \\
\text { cierta función pragmática }\end{array}$ & Sí & Sí-no & Sí \\
\hline
\end{tabular}

A la pregunta de si los usos considerados aquí de di (tú) que pueden caracterizarse como marcadores del discurso se puede contestar que si se evita una categorización discreta, basada en el cumplimiento de una serie de condiciones necesarias y suficientes, y si se acepta en cambio la posibilidad de que se cumplan solo algunas de las condiciones y en grados diversos, tal como se defiende desde la teoría de los prototipos (Lakoff 1987; Cuenca y Hilferty 1999), es evidente que todos ellos pueden clasificarse como marcadores del discurso ${ }^{14}$.

13 Se trata, evidentemente, de una interpretación muy amplia de "conexión". Para un análisis bibliográfico general sobre lo que representa la conexión en la definición de los marcadores del discurso, puede consultarse Landone (2009: 84-88). Autores como Pons Bordería (1998) o Cuenca (2001) acuden, desde los planteamientos de la categorización no discreta de la teoría de los prototipos, al concepto de conexión para fundamentar la categoría de los conectores (Pons Bordería) o conectores parentéticos (Cuenca). Otros autores circunscriben la función de conexión solo a algunos elementos, precisamente aquellos que denomina conectores. Por ejemplo, Fuentes Rodríguez (2009: 12) distingue entre conectores (relacionan varios enunciados) y operadores (su ámbito se reduce a un solo enunciado).

14 Los marcadores del discurso suelen caracterizarse como una categoría "pragmática" (Landone 2009: 76) o funcional (Pons Bordería 2000: 201) en la que se integran unidades muy diversas que, en ocasiones, cumplen la 
Teniendo en cuenta las propiedades recogidas en la tabla, di (tú) que de intensificación y di (tú) que hipotético son los que más se aproximan al prototipo de marcador del discurso. De hecho, la mayor parte de las propiedades que no se cumplen son consecuencia de las restricciones formales que impone la presencia de la conjunción que.

En cambio, di (tú) que con valor irónico se encuentra en una posición intermedia. Presenta un menor grado de fijación formal y de idiomaticidad que los otros dos valores. En su caso se podría hablar de "fórmula semiidiomática", en el sentido de que ese nuevo valor está basado en el significado original de la fórmula, significado que de alguna manera sigue latente ${ }^{15}$. Al haber una pervivencia del significado conceptual ligado al verbo decir, se aparta de la imagen prototípica de los marcadores discursivos como formas con significado exclusivamente procedimental.

En definitiva, di (tú) que como inductor de ironía presenta rasgos que lo aproximan a los procedimientos gramaticalizados y a los que se basan en el léxico. Se encuentra, por tanto, en un punto intermedio dentro del continuum léxico-gramática. Aunque no está plenamente gramaticalizado, se siente ya como un procedimiento ligado al cumplimiento de una función pragmático-discursiva $\mathrm{y}$, desde este planteamiento, puede considerarse representante, aunque periférico, de la categoría.

\section{CONCLUSIONES}

Nuestro estudio muestra que no se puede establecer una separación radical entre los procedimientos basados en el léxico y aquellos otros que se fundamentan en la gramática. Hay un trasvase natural entre ellos. Como apunta P. J. Hopper (1987) con su hipótesis de la gramática emergente, la gramática está siempre en proceso de construcción. Lo que comienza siendo una pauta de codificación de naturaleza léxica termina convirtiéndose en una nueva pauta de codificación, pero esta vez basada en la gramática. Aplicando esto a la aparición de ciertos marcadores del discurso, la teoría de la gramaticalización nos ha enseñado que este proceso de cambio es de naturaleza gradual y que tiene motivaciones pragmáticas (Hopper 1991; Hopper y Traugott 1993; Traugott 1995a y 1995b; Brinton 1996 y 2007; Company 2004; Alvarado Ortega y Ruiz Gurillo 2011).

En el caso de di (tú) que, como consecuencia de los cambios formales y semánticos verificados en su evolución, esta construcción mira cada vez más a las operaciones constitutivas del discurso y se borra de forma progresiva la referencia a otro acto de enunciación futuro. En otras palabras, deja de contribuir al significado conceptual del enunciado para incorporarse al ámbito de las expresiones que ayudan al procesamiento de lo que se comunica (Blakemore 1992: 149-150; Wilson y Sperber 1993). Desde un punto de vista gramatical, esto supone que lo que era una forma verbal de imperativo seguida de una completiva se ha ido transformando en diversos marcadores discursivos que afectan al conjunto de la proposición que se quiere comunicar. Semánticamente, lo que era una

función de marcación del discurso. Tienen, pues, una naturaleza procedimental. En la categorización pragmática no se puede hablar de criterios discretos, sino de un continuum escalar en el que podemos encontrar tanto realizaciones prototípicas como periféricas.

15 Según Zuluaga (1980: 134), en las expresiones semiidiomáticas "los significados de los componentes inciden en el sentido global de la expresión, pero este no se identifica, sin más, con el literal-regular”. 
inferencia pragmática sobre la verdad o falsedad de la antigua completiva ${ }^{16}$ (Si te preguntan, di que estás enfermo [y así sabrán la verdad] / Si te preguntan, di que estás enfermo [y asi se confiarán]) ha pasado a integrarse a la información codificada. Tal componente modal se acaba utilizando con diversos fines comunicativos: para intensificar un acto de habla en el que se transmite una información que se asume como verdadera o posiblemente verdadera (usos intensificadores), para realzar una información verdadera frente a una alternativa contraria (usos irónicos) o para destacarla en un mundo alternativo frente a otras opciones (usos hipotéticos) ${ }^{17}$.

\section{REFERENCIAS BIBLIOGRÁFICAS}

Albelda Marco, M. (2007). La intensificación como categoría pragmática: revisión y propuesta. Frankfurt: Peter Lang.

Alvarado Ortega, M. B. y L. Ruiz Gurillo (2011). "Un acercamiento fraseológico a desde luego", RILCE, 27-2, pp. 305-320.

Bazzanella, C. et alii (1991). "Scalar dimensions of illocutionary force". En Žagar, I. (ed.). Speech acts: fiction or reality? Proceedings of the International Conference, Ljubljana, November 15, 1990. Ljubljana: Inštitut za družbene vede, pp. 63-76.

Beinhauer, W. (1968). El español coloquial. Madrid: Gredos.

Blakemore, D. (1992). Understanding Utterances. An Introduction to Pragmatics. Oxford/Cambridge (Massachusetts): Blackwell.

Brinton, L. J. (1996). Pragmatic markers in English. Grammaticalization and discourse functions. Berlin: Mouton/De Gruyter.

Brinton, L. J. (2007). "The development of I mean: Implications for Historical Pragmatics". En Fitzmaurice, S.M. e I. Taavitsainen (dirs.). Methods in Historical Pragmatics. Berlin: Mouton de Gruyter, pp. 37-80.

Briz, A. (1996). "Los intensificadores en la conversación coloquial”. En Briz, A., S. Pons y J. Portolés (eds.). Pragmática y gramática del español hablado. Actas del II Simposio sobre análisis del discurso oral. Zaragoza: Pórtico Libros, pp. 13-36.

Briz, A. (1998). El español coloquial en la conversación. Esbozo de pragmagramática. Barcelona: Ariel.

Briz, A. et alii (coords.) (2008). Diccionario de partículas del español: http://www.dpde.es (10-122018).

Briz, A. y Grupo Val.Es.Co (2003). "Un sistema de unidades para el estudio del lenguaje coloquial”, Oralia, 6, pp. 7-61.

Caffi, C. (1999). “On mitigation”, Journal of Pragmatics, 31, pp. 881-909.

Company, C. (2004). “¿Gramaticalización o desgramaticalización? Reanálisis y subjetivización de verbos como marcadores discursivos en la historia del español”, RFE, LXXXIV, pp. 29-66.

Corpas, G. (1996). Manual de fraseología española. Madrid: Gredos.

Cortés, L. y M. Camacho (2005). Unidades de segmentación y marcadores del discurso. Elementos esenciales en el procesamiento discursivo. Madrid: Arco Libros.

Cuenca, M. J. (2001). "Los conectores parentéticos como categoría gramatical”, Lingüistica Española Actual, XXIII, 2, pp. 211-235.

Cuenca, M. J. y J. Hilferty (1999). Introducción a la lingüística cognitiva. Barcelona: Ariel.

16 Decir no es un verbo modal, sino enunciativo y todos los juicios modales que se puedan aplicar al contenido proposicional se determinan contextualmente.

17 Téngase en cuenta que los usos de di (tú) que que no incorporan ningún tipo de contenido asertivo son realmente extraños. 
Fernández Bernárdez, C. (2002). Expresiones metalingüisticas con el verbo decir. A Coruña: Universidade da Coruña.

Fuentes Rodríguez, C. (2009). Diccionario de conectores y operadores del español. Madrid: Arco Libros.

Grande Alija, F. J. (2013). "El imperativo di en las rutinas discursivas: modalidad, discurso y gramática", Verba, 40, pp. 157-202.

Grande Alija, F. J. (2014). "Creer y no creer: modalidad apelativa y aserción en el lenguaje”, Estudios Filológicos, 54, pp. 85-110.

Grande Alija, F. J. (2016). "Imperativo, subjuntivo y el espacio desiderativo-apelativo", Círculo de lingüística aplicado a la comunicación, 67, pp. 167-211.

Guillén Escamilla, J. E. (2018). "Hacia una caracterización funcional de fijarse como marcador del discurso", Pragmalingüística, 26, pp. 131-147. DOI: http://dx.doi.org/10.25267/Pragmalinguistica.2018.i26.07.

Hopper, P. J. (1987). “Emergent grammar”, Berkeley Linguistic Society, 13, pp. 139-157.

Hopper, P. J. (1991). “On some principles of grammaticization”. En Traugott, E. C. y B. Heine (eds.). Approaches to Grammaticalization, vol. 1. Amsterdam: John Benjamins, pp. 17-35.

Hopper, P. J. y E. Traugott (1993). Grammaticalization. Cambridge: Cambridge University Press.

Lakoff, G. (1987). Women, Fire and Dangerous Things. What Categories Reveal about the Mind. Chicago: University of Chicago Press.

Landone, E. (2009). Los marcadores del discurso y cortesía verbal en español. Bern: Peter Lang.

Martín Zorraquino, M. A. y J. Portolés Lázaro (1999): "Los marcadores del discurso". En Bosque, I. y V. Demonte (dirs.). Gramática descriptiva del español. Entre oración y discurso. Morfología. Vol. 3. Madrid: Espasa-Calpe, pp. 4051-4213.

Meyer-Hermann, R. (1988). “Atenuación e intensificación (análisis pragmático de sus formas y funciones en español hablado)”, Anuario de Estudios Filológicos, 11, pp. 275-290.

Mihatsch, W. y M. Albelda Marco (2016). "Introducción. La atenuación y la intensificación desde una perspectiva semántico-pragmática”, Revista internacional de lingüística iberoamericana, 27, pp. 7-18.

Moliner, M. (2004). Diccionario del uso del español. Madrid: Gredos.

Morgan, J. L. (1978). "Two types of convention in indirect speech acts". En Cole, P. (dir.). Syntax and Semantics 9. Pragmatics. New York: Academic Press, pp. 261-280.

Pons Bordería, S. (1998). Conexión y conectores. Estudio de su relación en el registro informal de la lengua. Valencia: Universidad de Valencia.

Pons Bordería, S. (2000). “Los conectores”. En Briz, A. y Grupo Val.Es.Co. (dirs.). ¿Cómo se comenta un texto coloquial? Barcelona: Ariel, pp. 193-220.

Real Academia Española (2014). Diccionario de la lengua española, vigésima tercera edición. Madrid: Espasa.

Ruiz Gurillo, L. (1997). Aspectos de fraseología teórica española. Valencia: Universidad de Valencia.

Ruiz Gurillo, L. (1998). La fraseología del español coloquial. Barcelona: Ariel.

Ruiz Gurillo, L. (2001). Las locuciones en español actual. Madrid: Arco Libros.

Santos Río, L. (2003). Diccionario de partículas. Salamanca: Luso-Española de Ediciones.

Sbisà, M. (2001): "Illocutionary force and degrees of strength in language use", Journal of Pragmatics, 33, pp. 1791-1814.

Seco, M., O. Andrés y G. Ramos (1999). Diccionario del español actual. Madrid: Aguilar.

Seco, M., O. Andrés y G. Ramos (2004). Diccionario fraseológico documentado del español actual. Locuciones y modismos españoles. Madrid: Aguilar.

Steel, B. (1976). A Manual of Colloquial Spanish. Madrid: Sociedad General Española de Librería.

Traugott, E. C. (1995a). "Subjectification in grammaticalization". En Wright, S. y D. Stein (dirs.). Subjectivity and Subjectivization. Cambridge: Cambridge University Press, pp. 31-54. 
Traugott, E. C. (1995b). "The role of grammaticalization in the development of discourse markers", comunicación presentada en el ICHL XII (Manchester): https://web.stanford.edu/ traugott/papers/ discourse.pdf (3-9-2018).

Wilson, D. y D. Sperber (1993). "Linguistic form and relevance", Lingua, 90, pp. 1-25.

Zuluaga, A. (1980). Introducción al estudio de las expresiones fijas. Frankfurt am Main: Lang.

\section{Corpus consultados}

Olmo Flecha, Ma . I. del (2005). La conversación telefónica: cortesía femenina y masculina. El corpus, vol. II (Tesis doctoral). León: Universidad de León.

PRESEEA (2014-). Corpus del Proyecto para el estudio sociolingüistico del español de España y de América. Alcalá de Henares: Universidad de Alcalá: http://preseea.linguas.net (8-11-2018).

Real Academia Española. Corpus diacrónico del español (CORDE): http://www.rae (27-11-2018).

Real Academia Española. Corpus de referencia del español actual (CREA): http://www.rae (6-112018).

Real Academia Española. Corpus del español del siglo XXI (CORPES): http://www.rae (19-11-2018). 